\title{
Cilia in the choroid plexus: their roles in hydrocephalus and beyond
}

\author{
Keishi Narita and Sen Takeda * \\ Department of Anatomy and Cell Biology, Interdisciplinary Graduate School of Medicine and Engineering, University of Yamanashi, Chuo, Yamanashi, Japan
}

\author{
Edited by: \\ Carlos Spuch, Institute of \\ Biomedical Research of Vigo (IBIV), \\ Spain \\ Reviewed by: \\ Hari S. Sharma, Uppsala University, \\ Sweden \\ Hariharasubramanian \\ Ramakrishnan, State University of \\ New York, USA \\ *Correspondence: \\ Sen Takeda, Department of \\ Anatomy and Cell Biology, \\ Interdisciplinary Graduate School of \\ Medicine and Engineering, \\ University of Yamanashi, 1110, \\ Shimo-Kateau, Chuo, Yamanashi \\ 409-3898, Japan \\ e-mail: stakeda@yamanashi.ac.jp
}

Cilia are whip-like projections that are widely conserved in eukaryotes and function as a motile propeller and/or sensory platform to detect various extracellular stimuli. In vertebrates, cilia are ubiquitously found in most cells, showing structural and functional diversities depending on the cell type. In this review, we focus on the structure and function of cilia in choroid plexus epithelial cells (CPECS). CPECs form one or two dozen non-motile 9+0 cilia, which display transient acquisition of motility during development. Genetic malfunction of cilia can lead to failure of multiple organs including the brain. Especially, several groups have demonstrated that the defects in CPEC cilia cause the communicating form of hydrocephalus. In order to elucidate the molecular mechanisms underlying the hydrocephalus, we have previously demonstrated that the cilia possess an NPFF receptor for autocrine signaling to regulate transepithelial fluid transport. In this perspective, we also discuss the potential involvement of cilia in the other aspects of choroid plexus functions, such as the regulation of brain development and neuroinflammation.

Keywords: cilia, diversity, hydrocephalus, multiciliogenesis, cerebrospinal fluid

\section{OVERVIEW OF VERTEBRATE CILIA}

Cilia are hair-like projections on the cell surface with a diameter of $\sim 250 \mathrm{~nm}$ and various lengths of typically $5-10 \mu \mathrm{m}$ (Figure 1A). Their structure is supported and anchored to the cell by characteristic cytoskeletal scaffolds called the axoneme and basal body in which doublet and triplet microtubules, respectively, are radially arranged with nine-fold symmetry. Cilia are widely conserved across eukaryotic species, and in many unicellular organisms, their active vibration is necessary for propelling the cell. In vertebrates, cilia have been observed with various characteristics, such as length, motility, and number per cell, depending on the tissues and cell type including neurons and glia in the brain (Gerdes et al., 2009; Louvi and Grove, 2011; Takeda and Narita, 2012).

For example, ependyma (ependymocytes) lining brain ventricles form hundreds of motile cilia to circulate the cerebrospinal fluid (CSF). The axoneme of this ciliary subtype has a central pair of singlet microtubules (termed " $9+2$ "), and is heavily equipped with axonemal dyneins and their regulatory complexes, which collectively drive the back-and-forth movement of cilia (Figure 1B, left; Afzelius, 2004; Lindemann and Lesich, 2010). In contrast, most neurons and glia possess solitary non-motile cilia called primary cilia. Their axoneme has no central pair and is termed " $9+0$ " (Figure 1B, right). Compared with 9+2 cilia (Heuser et al., 2009; Pigino et al., 2011), the structural details have been poorly resolved in $9+0$ cilia (Gilliam et al., 2012). Although most primary cilia appear to lack axonemal dyneins and are non-motile except for nodal cilia (Takeda et al., 1999; Hirokawa et al., 2009), they harbor various cell signaling receptors and mediators to detect and process mechanical stress or chemical stimuli such as Sonic hedgehog and platelet-derived growth factor (Praetorius and Spring, 2001; Corbit et al., 2005; Schneider et al., 2005; Yoshimura et al., 2011; Briscoe and Thérond, 2013; Su et al., 2013). The outer segment of photoreceptors in the retina, where photosensitive rhodopsins are packed in a series of membranous discs, is a specialized form of primary cilia (Gilliam et al., 2012).

Genetic defects leading to ciliary malfunctions cause disorders with clinically variable phenotypes. Such disorders are called ciliopathies and include primary ciliary dyskinesia, polycystic kidney disease, Leber congenital amaurosis, nephronophthisis, Senior-Løken syndrome, Joubert syndrome, Bardet-Biedl syndrome, and Meckel Gruber syndrome (Novarino et al., 2011). These ciliopathies are often associated with brain diseases such as neural tube defects, cerebellar hypoplasia, mental retardation, and hydrocephalus.

\section{BIOGENESIS OF CILIA}

Numerous studies using various model organisms, such as green algae, worms, fish, frogs, and mice, as well as human subjects, have founded the principle of ciliogenesis as recapitulated below. This information also provides the basis to understand ciliopathies.

The biogenesis of cilia is initiated by assembling axonemes and docking ciliary membrane vesicles to the distal end of the basal body. A specialized transport system called "intraflagellar transport" (IFT) carries tubulin and other materials along the axoneme (Rosenbaum and Witman, 2002). IFT facilitates 


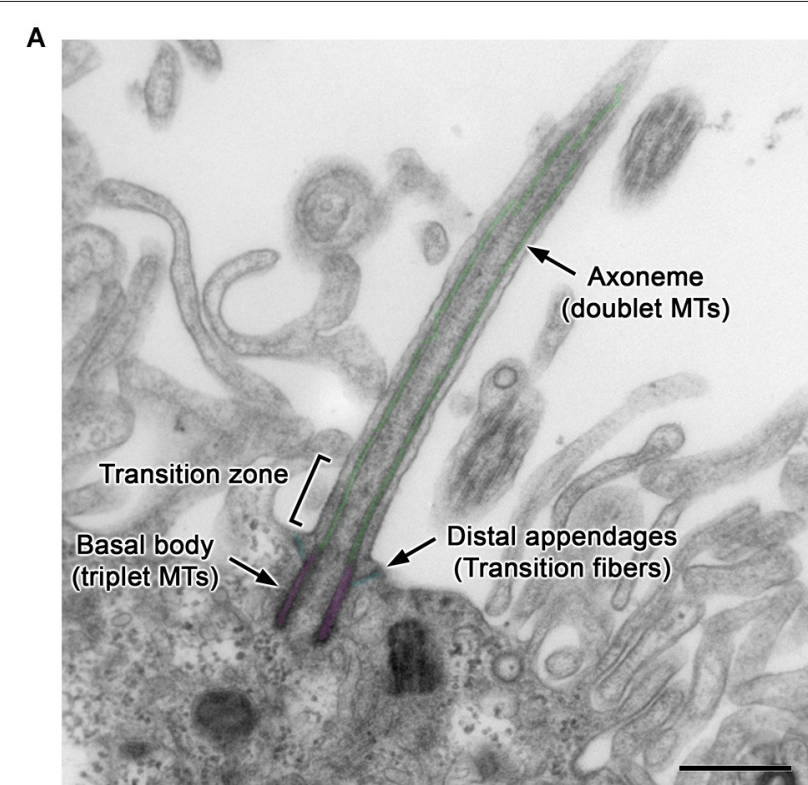

B

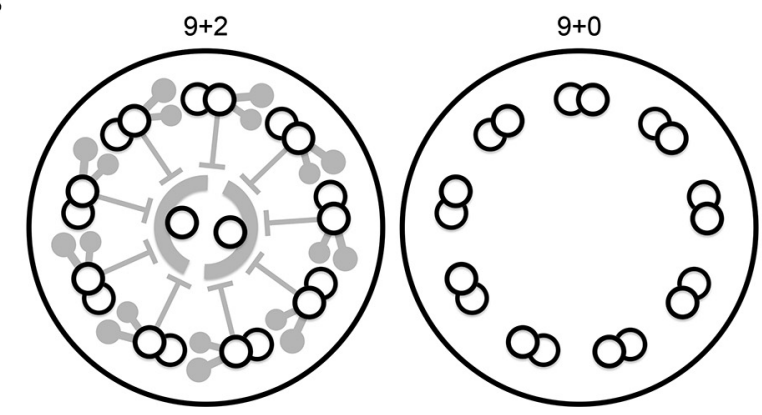

FIGURE 1 | General structure of cilia. (A) A longitudinal section of a CPEC cilium. The cilium emerges from the microvilli-rich apical cell surface. The structure is supported by the axoneme and basal body (pseudo-colored in green and magenta, respectively). The distal appendage (cyan) connects to the basal body and cell membrane. In the transition zone, characteristic Y-shaped structures bridges the axoneme and ciliary membrane, which can be recognized in horizontal sections. MTs, microtubules. Bar, $500 \mathrm{~nm}$. (B) Schematics of transverse sections of motile $9+2$ and non-motile $9+0$ cilia.

microtubule motor proteins, kinesins and cytoplasmic dyneins, as well as IFT particles A and B, which mediate cargo attachment to the motors. For docking to the ciliary membrane, structural components of the appendages on the basal body are required (Tanos et al., 2013; Veleri et al., 2014). Furthermore, the regulation of molecules entering and leaving cilia is mediated by several other systems including the BBSome (Nachury et al., 2010). A molecular sieve and septin ring at the ciliary base also restrict simple diffusion of soluble and membrane proteins, respectively (Hu et al., 2010; Breslow et al., 2013; Lin et al., 2013).

The basal body of a primary cilium is a modified mother centriole. When cells enter the cell cycle, the primary cilium is shortened, and the basal body is detached from the cell surface to function as a microtubule-organizing center (Paridaen et al., 2013). On the other hand, the basal bodies of multiciliated cells are generated explosively de novo at intracellular foci called deuterosomes by the so-called acentriolar pathway (Klos Dehring et al., 2013). Foxj1 is one of the transcription factors that act as a master regulator of multiciliogenesis (Thomas et al., 2010).

\section{CILIA IN CHOROID PLEXUS EPITHELIAL CELLS}

The choroid plexus is a highly undulating and vascularized tissue that protrudes into brain ventricles. Its epithelium consists of choroid plexus epithelial cells (CPECs) that produce CSF with high efficiency (Damkier et al., 2013). In addition, the choroid plexus epithelium secretes ligands that are important for brain physiology, and regulates protein diffusion and leukocyte infiltration from systemic circulation (Redzic et al., 2005; Reboldi et al., 2009; Shechter et al., 2013). Because CPECs are derived from the dorsal neuroepithelium and form a continuous monolayer with ependyma, they are sometimes described as choroidal or modified ependyma. However, CPECs and ependyma are distinct in many aspects, which is also the case for cilia.

As described above, mature ependyma form hundreds of motile $9+2$ cilia that beat in a concerted manner to circulate CSF. In mouse, the multiciliogenesis initiates after birth and requires about 2 weeks for full maturation (Figure 2; Spassky et al., 2005). In contrast, CPECs form one or two dozen nonmotile 9+0 cilia (Narita et al., 2010). Ciliogenesis in CPECs occurs shortly after the choroid plexus primordia begins to bud during organogenesis (Figure 2; Nonami et al., 2013). In addition, CPEC cilia exhibit transient motility around the perinatal period, yet a low beating frequency, small amplitude, and random orientation are all unfavorable to generate directional CSF flow (Narita et al., 2012). The motility peaks at around the day of birth and declines progressively during the following 2 weeks. While both CPECs and ependyma may share a common, FOXJ1dependent mechanism to initiate multiciliogenesis (Lim et al., 1997; Narita et al., 2012), their cilia show different characteristics. This observation is intriguing from the viewpoint of the current principle.

Genetically modified mouse models have also shown differences in the mechanism of ciliary formation and/or the maintenance of cilia in CPECs and ependyma. In a knockout mouse for Celsr2, an ortholog of the planar cell polarity gene Flamingo, an impairment of ciliogenesis is observed in ependyma but not in CPECs (Tissir et al., 2010). Similarly, forced expression of the PAC1 (phosphatase of activated cells 1) receptor, a G protein-coupled receptor that is predominantly expressed in the central nervous system (CNS) and selectively activated by pituitary adenylate cyclase-activating polypeptide, causes severe hydrocephalus associated with disorganization of ependymal cilia, while CPEC cilia are unaffected (Lang et al., 2006).

Regarding the unique function of CPEC cilia, several groups including ours have reported the potential involvement of CPEC cilia in the regulation of CSF production. Analysis of CPEC cilia in relation to the hydrocephalus was first described by Yoder et al. (Banizs et al., 2005). In the Ift88 $8^{\text {Tg737Rpw }}$ mouse that has defects in IFT88 expression and function, Banizs et al. observed a communicating form of the hydrocephalus at neonatal periods, 


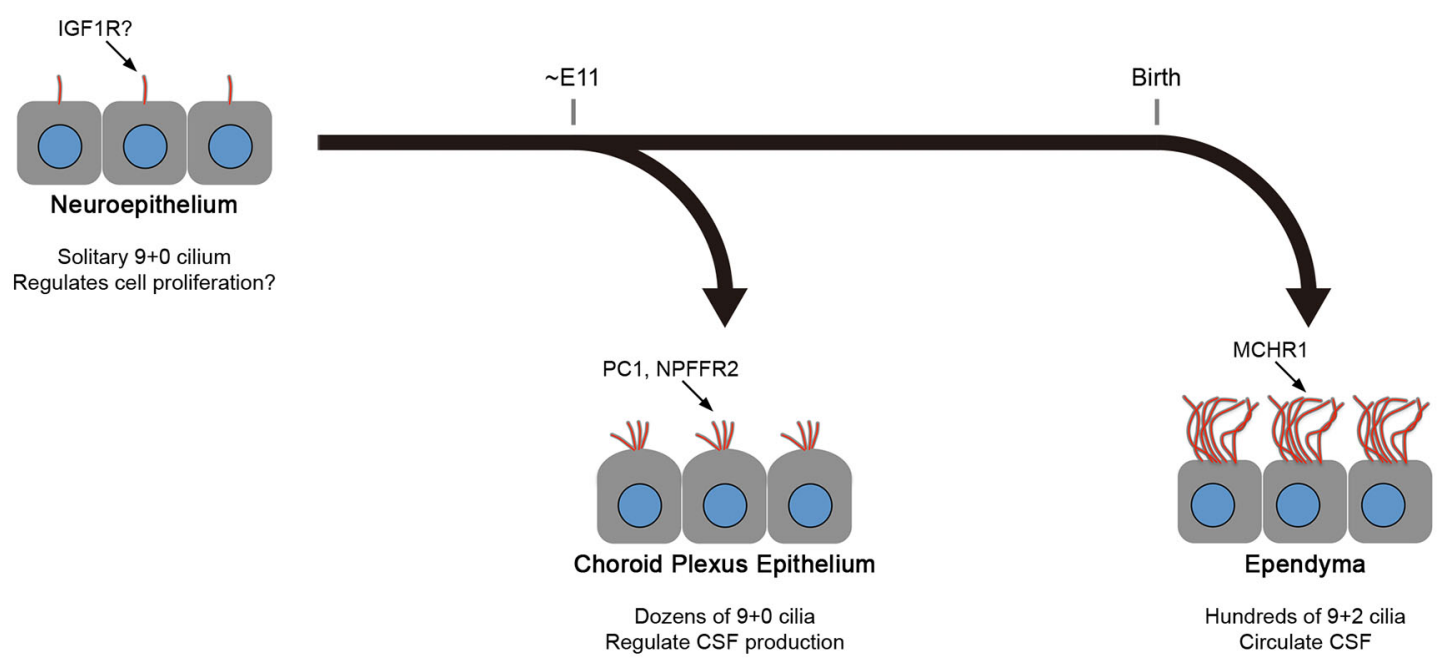

FIGURE 2 | Differences between CPEC and ependymal cilia. The formation of multiple cilia in CPECs occurs shortly after the cells differentiate from the neuroepithelium during organogenesis (about embryonic day 11 in mice). The cilia exhibit transient motility during the perinatal period, which peaks at the day of birth, and eventually become non-motile. However, ependyma undergo multiciliogenesis after birth to establish hundreds of motile cilia in 2 weeks. The beating orientation is aligned at both cellular and tissue levels by planar cell polarity signaling. In both cell types, multiciliogenesis is associated with the induction of transcription factors, FOXJ1 and RFX3. Ciliary localization of indicated molecules in neuroepithelium, choroid plexus epithelium, and ependyma are reported or implicated in Lehtinen et al. (2011), (Banizs et al., 2005; Wodarczyk et al., 2009; Narita et al., 2010), and Conductier et al. (2013), respectively. Knockout mice lacking general ciliogenesis genes, such as Ift88, Kif3a, and Bbs1,2,4, and 6, exhibit the communicating form of hydrocephalus. See text for details. when most ependyma lack mature motile cilia. During these stages, CPEC cilia show an accumulation of polycystin-1, the defects of which cause autosomal dominant polycystic kidney disease, in a bulb-like structure at the tip. This abnormal ciliary structure and protein localization coincide with an increase in cellular cAMP levels and aberrant regulation of intracellular $\mathrm{pH}$ and ion transport activities in CPECs (Banizs et al., 2005, 2007). Similarly, Wodarczyk et al. also described the ciliary localization of polycystin-1 in CPECs and ependyma (Wodarczyk et al., 2009). They generated ubiquitous or brain-specific $P k d 1$ knockout mice, which encodes polycystin-1, and observed hydrocephalus at perinatal periods in both mouse lines.

We used a primary culture system for swine CPECs to analyze ciliary function and showed that deciliation by chloral hydrate increases both intracellular cAMP levels and basolateral-to-apical transepithelial fluid transcytosis, which is consistent with the above observations by Banizs et al. (Narita et al., 2010). We also demonstrated localization of neuropeptide FF receptor 2 on CPEC cilia, and its autoactivation downregulated cellular cAMP levels and fluid transcytosis. While the mechanism involves negative regulation of CSF production, we do not know whether there is a positive regulator or the production is sustained continuously, and only negative regulation controls the amount of CSF (Lindvall et al., 1978; Damkier et al., 2013). This point has to be addressed in the near future.

Swiderski et al. investigated the mechanism of ventriculomegaly that is common in ciliopathy models of $B b s 1, B b s 2$, Bbs4, and Bbs6 mutant mice (Swiderski et al., 2012). While ventriculomegaly is not associated with stenosis of the cerebral aqueduct, ultrastructural abnormalities in the cilia of CPECs, ependyma, and some circumventricular organs are observed consistently in these mutant mice at various ages. The previous study also concluded that a loss of regulation in CSF production is one of the possible mechanisms underlying the pathology.

Recently, Liu et al. generated a conditional knockout of Kif3a in cranial neural crest cells, using a Wnt1 promoterdriven Cre recombinase (Liu et al., 2014). KIF3A is a kinesin motor protein involved in ciliogenesis and plays a crucial role in the determination of left-right asymmetry of the body (Takeda et al., 1999). The genetically modified mice exhibited ciliopathy phenotypes of craniofacial anomalies and hydrocephalus. Regarding the hydrocephalus, the authors observed a dramatic dilation of the lateral and third ventricles in E16.5 embryo. Having confirmed the Wnt $1^{\text {cre }}$ expression in E16.5 choroid plexuses, they concluded that the hydrocephalus is due to overproduction of CSF (Liu et al., 2014).

\section{FUTURE PERSPECTIVES}

The above studies implicate defects in CPEC cilia as a cause of the communicating form of hydrocephalus. However, reports by Durand et al. suggest additional mechanisms. They generated mice deficient for $\mathrm{Rfx} 3$, a transcription factor that regulates ciliogenesis, and demonstrated marked inhibition of ciliogenesis in both CPECs and ependyma, which is associated with the communicating form of hydrocephalus (Baas et al., 2006; El Zein et al., 2009) and in agreement with the above studies. Interestingly, the authors also observed marked choroid plexus hypogenesis in the knockout mouse (Baas et al., 2006; El Zein et al., 2009). Because CPECs synthesize and secrete various growth factors and signaling molecules for brain development 
(Redzic et al., 2005; Reboldi et al., 2009; Shechter et al., 2013), a reduction in the mass of choroid plexus tissue may lead to brain abnormalities other than hydrocephalus. Indeed, the authors later demonstrated that the $R f x 3$ knockout mouse also exhibits corpus callosum agenesis (Benadiba et al., 2012), although the significance of choroid plexus hypogenesis in this phenotype is unclear.

A growing body of evidence suggests that the choroid plexus functions as a selective and educative gate for circulating immune cells in the immune surveillance of the CNS to resolve neuroinflammation under pathological conditions (Schwartz and Baruch, 2014). The apical surface of CPECs is the site where immune cells reside even under physiological conditions. These cells were initially described as epiplexus cells or Kolmer cells (Ling et al., 1998), and are now recognized as dendritic cells and macrophages that function as local antigen-presenting cells (Ransohoff and Engelhardt, 2012). When activated by inflammatory cytokines, CPECs upregulate their expression of integrin receptors to promote immune cells entering the CNS (Engelhardt et al., 2001; Shechter et al., 2013). Because of the physical proximity, it is possible that CPEC cilia make direct contact with these immune cells and/or receive chemical substances secreted by them, thereby participating in the regulation of choroid plexus functions in response to neuroinflammation.

Recently, we performed proteomic analysis of CPEC cilia from swine and identified $>800$ proteins (Narita et al., 2012). Among them, $45 \%$ were shared with the proteome of the $9+0$ photoreceptor outer segment and $18 \%$ were shared with the proteome of $9+2$ cilia and flagella. Among the remaining $37 \%$ of the proteins including the CPEC-specific ciliome subset, various signaling molecules were enriched. Functional analysis of these proteins will clarify the role of CPEC cilia in more detail and their link to brain disorders.

According to the traditional view, CPECs have been regarded as solely responsible for the production of CSF. However, based on our current understanding of CSF production, we should reinterpret or re-evaluate the traditional views of CSF homeostasis (Iliff et al., 2012), which are not necessarily obsolete or invalid. In this regard, the cilia in CPECs may have various unknown functions that are related to maintenance of brain homeostasis. Therefore, cilia in the brain ventricular system play important biological roles in neurophysiology and may further advance our understanding of brain functions.

\section{ACKNOWLEDGMENTS}

This review was supported by Grants-in-Aid for Scientific Research (C) from the Ministry of Education, Culture, Sports, Science and Technology of Japan to ST (19590188) and KN (26440094).

\section{REFERENCES}

Afzelius, B. A. (2004). Cilia-related diseases. J. Pathol. 204, 470-477. doi: 10. 1002/path. 1652

Baas, D., Meiniel, A., Benadiba, C., Bonnafe, E., Meiniel, O., Reith, W., et al. (2006). A deficiency in RFX3 causes hydrocephalus associated with abnormal differentiation of ependymal cells. Eur. J. Neurosci. 24, 1020-1030. doi: 10. 1111/j.1460-9568.2006.05002.x
Banizs, B., Komlosi, P., Bevensee, M. O., Schwiebert, E. M., Bell, P. D., and Yoder, B. K. (2007). Altered $\mathrm{pH}(\mathrm{i})$ regulation and $\mathrm{Na}(+) / \mathrm{HCO} 3(-)$ transporter activity in choroid plexus of cilia-defective Tg737(orpk) mutant mouse. Am. J. Physiol. Cell Physiol. 292, C1409-C1416. doi: 10.1152/ajpcell.00408.2006

Banizs, B., Pike, M. M., Millican, C. L., Ferguson, W. B., Komlosi, P., Sheetz, J., et al. (2005). Dysfunctional cilia lead to altered ependyma and choroid plexus function and result in the formation of hydrocephalus. Development 132, 53295339. doi: 10.1242/dev.02153

Benadiba, C., Magnani, D., Niquille, M., Morle, L., Valloton, D., Nawabi, H., et al. (2012). The ciliogenic transcription factor RFX3 regulates early midline distribution of guidepost neurons required for corpus callosum development. PLoS Genet. 8:e1002606. doi: 10.1371/journal.pgen.1002606

Breslow, D. K., Koslover, E. F., Seydel, F., Spakowitz, A. J., and Nachury, M. V. (2013). An in vitro assay for entry into cilia reveals unique properties of the soluble diffusion barrier. J. Cell Biol. 203, 129-147. doi: 10.1083/jcb.201212024

Briscoe, J., and Thérond, P. P. (2013). The mechanisms of Hedgehog signalling and its roles in development and disease. Nat. Rev. Mol. Cell Biol. 14, 416-429. doi: 10.1038/nrm3598

Conductier, G., Brau, F., Viola, A., Langlet, F., Ramkumar, N., Dehouck, B., et al. (2013). Melanin-concentrating hormone regulates beat frequency of ependymal cilia and ventricular volume. Nat. Neurosci. 16, 845-847. doi: 10.1038/nn.3401

Corbit, K. C., Aanstad, P., Singla, V., Norman, A. R., Stainier, D. Y., and Reiter, J. F. (2005). Vertebrate Smoothened functions at the primary cilium. Nature 437, 1018-1021. doi: 10.1038/nature04117

Damkier, H. H., Brown, P. D., and Praetorius, J. (2013). Cerebrospinal fluid secretion by the choroid plexus. Physiol. Rev. 93, 1847-1892. doi: 10. 1152/physrev.00004.2013

El Zein, L., Ait-Lounis, A., Morlé, L., Thomas, J., Chhin, B., Spassky, N., et al. (2009). RFX3 governs growth and beating efficiency of motile cilia in mouse and controls the expression of genes involved in human ciliopathies. J. Cell Sci. 122, 3180-3189. doi: 10.1242/jcs.048348

Engelhardt, B., Wolburg-Buchholz, K., and Wolburg, H. (2001). Involvement of the choroid plexus in central nervous system inflammation. Microsc. Res. Tech. 52, 112-129. doi: 10.1002/1097-0029(20010101)52:1<112::AID-JEMT13>3.0. $\mathrm{CO} ; 2-5$

Gerdes, J. M., Davis, E. E., and Katsanis, N. (2009). The vertebrate primary cilium in development, homeostasis and disease. Cell 137, 32-45. doi: 10.1016/j.cell. 2009.03.023

Gilliam, J. C., Chang, J. T., Sandoval, I. M., Zhang, Y., Li, T., Pittler, S. J., et al. (2012). Three-dimensional architecture of the rod sensory cilium and its disruption in retinal neurodegeneration. Cell 151, 1029-1041. doi: 10.1016/j.cell.2012.10.038

Heuser, T., Raytchev, M., Krell, J., Porter, M. E., and Nicastro, D. (2009). The dynein regulatory complex is the nexin link and a major regulatory node in cilia and flagella. J. Cell Biol. 187, 921-933. doi: 10.1083/jcb.200908067

Hirokawa, N., Tanaka, Y., and Okada, Y. (2009). Left-right determination: involvement of molecular motor KIF3, cilia and nodal flow. Cold Spring Harb Perspect. Biol. 1:a000802. doi: 10.1101/cshperspect.a000802

Hu, Q., Milenkovic, L., Jin, H., Scott, M. P., Nachury, M. V., Spiliotis, E. T., et al. (2010). A septin diffusion barrier at the base of the primary cilium maintains ciliary membrane protein distribution. Science 329, 436-439. doi: 10. 1126/science. 1191054

Iliff, J. J., Wang, M., Liao, Y., Plogg, B. A., Peng, W., Gundersen, G. A., et al. (2012). A paravascular pathway facilitates CSF flow through the brain parenchyma and the clearance of interstitial solutes, including amyloid beta. Sci. Transl. Med. 4:147ra111. doi: 10.1126/scitranslmed.3003748

Klos Dehring, D. A., Vladar, E. K., Werner, M. E., Mitchell, J. W., Hwang, P., and Mitchell, B. J. (2013). Deuterosome-mediated centriole biogenesis. Dev. Cell 27, 103-112. doi: 10.1016/j.devcel.2013.08.021

Lang, B., Song, B., Davidson, W., MacKenzie, A., Smith, N., McCaig, C. D., et al. (2006). Expression of the human PAC1 receptor leads to dose-dependent hydrocephalus-related abnormalities in mice. J. Clin. Invest. 116, 1924-1934. doi: $10.1172 /$ jci2 27597

Lehtinen, M. K., Zappaterra, M. W., Chen, X., Yang, Y. J., Hill, A. D., Lun, M., et al. (2011). The cerebrospinal fluid provides a proliferative niche for neural progenitor cells. Neuron 69, 893-905. doi: 10.1016/j.neuron.2011.01.023

Lim, L., Zhou, H., and Costa, R. H. (1997). The winged helix transcription factor HFH-4 is expressed during choroid plexus epithelial development in the mouse embryo. Proc. Natl. Acad. Sci. U S A 94, 3094-3099. doi: 10.1073/pnas.94.7. 3094 
Lin, Y. C., Niewiadomski, P., Lin, B., Nakamura, H., Phua, S. C., Jiao, J., et al. (2013). Chemically inducible diffusion trap at cilia reveals molecular sieve-like barrier. Nat. Chem. Biol. 9, 437-443. doi: 10.1038/nchembio. 1252

Lindemann, C. B., and Lesich, K. A. (2010). Flagellar and ciliary beating: the proven and the possible. J. Cell Sci. 123, 519-528. doi: 10.1242/jcs.051326

Lindvall, M., Edvinsson, L., and Owman, C. (1978). Sympathetic nervous control of cerebrospinal fluid production from the choroid plexus. Science 201, 176-178. doi: 10.1126/science.663649

Ling, E. A., Kaur, C., and Lu, J. (1998). Origin, nature and some functional considerations of intraventricular macrophages, with special reference to the epiplexus cells. Microsc. Res. Tech. 41, 43-56. doi: 10.1002/(sici)10970029(19980401)41:1<43::aid-jemt5>3.3.co;2-d

Liu, B., Chen, S., Johnson, C., and Helms, J. A. (2014). A ciliopathy with hydrocephalus, isolated craniosynostosis, hypertelorism and clefting caused by deletion of Kif3a. Reprod. Toxicol. 48, 88-97. doi: 10.1016/j.reprotox.2014. 05.009

Louvi, A., and Grove, E. A. (2011). Cilia in the CNS: the quiet organelle claims center stage. Neuron 69, 1046-1060. doi: 10.1016/j.neuron.2011.03.002

Nachury, M. V., Seeley, E. S., and Jin, H. (2010). Trafficking to the ciliary membrane: how to get across the periciliary diffusion barrier? Annu. Rev. Cell Dev. Biol. 26, 59-87. doi: 10.1146/annurev.cellbio.042308.113337

Narita, K., Kawate, T., Kakinuma, N., and Takeda, S. (2010). Multiple primary cilia modulate the fluid transcytosis in choroid plexus epithelium. Traffic 11, 287301. doi: 10.1111/j.1600-0854.2009.01016.x

Narita, K., Kozuka-Hata, H., Nonami, Y., Ao-Kondo, H., Suzuki, T., Nakamura, H., et al. (2012). Proteomic analysis of multiple primary cilia reveals a novel mode of ciliary development in mammals. Biol. Open 1, 815-825. doi: 10.1242/bio. 20121081

Nonami, Y., Narita, K., Nakamura, H., Inoue, T., and Takeda, S. (2013). Developmental changes in ciliary motility on choroid plexus epithelial cells during the perinatal period. Cytoskeleton (Hoboken) 70, 797-803. doi: 10. $1002 / \mathrm{cm} .21132$

Novarino, G., Akizu, N., and Gleeson, J. G. (2011). Modeling human disease in humans: the ciliopathies. Cell 147, 70-79. doi: 10.1016/j.cell.2011.09.014

Paridaen, J. T., Wilsch-Brauninger, M., and Huttner, W. B. (2013). Asymmetric inheritance of centrosome-associated primary cilium membrane directs ciliogenesis after cell division. Cell 155, 333-344. doi: 10.1016/j.cell.2013. 08.060

Pigino, G., Bui, K. H., Maheshwari, A., Lupetti, P., Diener, D., and Ishikawa, T. (2011). Cryoelectron tomography of radial spokes in cilia and flagella. J. Cell Biol. 195, 673-687. doi: 10.1083/jcb.201106125

Praetorius, H. A., and Spring, K. R. (2001). Bending the MDCK cell primary cilium increases intracellular calcium. J. Membr. Biol. 184, 71-79. doi: 10.1007/s00232001-0075-4

Ransohoff, R. M., and Engelhardt, B. (2012). The anatomical and cellular basis of immune surveillance in the central nervous system. Nat. Rev. Immunol. 12, 623635. doi: 10.1038/nri3265

Reboldi, A., Coisne, C., Baumjohann, D., Benvenuto, F., Bottinelli, D., Lira, S., et al. (2009). C-C chemokine receptor 6-regulated entry of TH-17 cells into the CNS through the choroid plexus is required for the initiation of EAE. Nat. Immunol. 10, 514-523. doi: 10.1038/ni.1716

Redzic, Z. B., Preston, J. E., Duncan, J. A., Chodobski, A., and SzmydyngerChodobska, J. (2005). The choroid plexus-cerebrospinal fluid system: from development to aging. Curr. Top. Dev. Biol. 71, 1-52. doi: 10.1016/s00702153(05)71001-2

Rosenbaum, J. L., and Witman, G. B. (2002). Intraflagellar transport. Nat. Rev. Mol. Cell Biol. 3, 813-825. doi: 10.1038/nrm952

Schneider, L., Clement, C. A., Teilmann, S. C., Pazour, G. J., Hoffmann, E. K., Satir, P., et al. (2005). PDGFRalphaalpha signaling is regulated through the primary cilium in fibroblasts. Curr. Biol. 15, 1861-1866. doi: 10.1016/j.cub.2005. 09.012
Schwartz, M., and Baruch, K. (2014). The resolution of neuroinflammation in neurodegeneration: leukocyte recruitment via the choroid plexus. EMBO J. 33, 7-22. doi: 10.1002/embj.201386609

Shechter, R., Miller, O., Yovel, G., Rosenzweig, N., London, A., Ruckh, J., et al. (2013). Recruitment of beneficial M2 macrophages to injured spinal cord is orchestrated by remote brain choroid plexus. Immunity 38, 555-569. doi: 10 . 1016/j.immuni.2013.02.012

Spassky, N., Merkle, F. T., Flames, N., Tramontin, A. D., García-Verdugo, J. M., and Alvarez-Buylla, A. (2005). Adult ependymal cells are postmitotic and are derived from radial glial cells during embryogenesis. J. Neurosci. 25, 10-18. doi: 10. 1523/jneurosci.1108-04.2005

Su, S., Phua, S. C., DeRose, R., Chiba, S., Narita, K., Kalugin, P. N., et al. (2013). Genetically encoded calcium indicator illuminates calcium dynamics in primary cilia. Nat. Methods 10, 1105-1107. doi: 10.1038/nmeth.2647

Swiderski, R. E., Agassandian, K., Ross, J. L., Bugge, K., Cassell, M. D., and Yeaman, C. (2012). Structural defects in cilia of the choroid plexus, subfornical organ and ventricular ependyma are associated with ventriculomegaly. Fluids Barriers CNS 9:22. doi: 10.1186/2045-8118-9-22

Takeda, S., and Narita, K. (2012). Structure and function of vertebrate cilia, towards a new taxonomy. Differentiation 83, S4-S11. doi: 10.1016/j.diff.2011. 11.002

Takeda, S., Yonekawa, Y., Tanaka, Y., Okada, Y., Nonaka, S., and Hirokawa, N. (1999). Left-right asymmetry and kinesin superfamily protein KIF3A: new insights in determination of laterality and mesoderm induction by kif3A-/- mice analysis. J. Cell Biol. 145, 825-836. doi: 10.1083/jcb.145.4.825

Tanos, B. E., Yang, H. J., Soni, R., Wang, W. J., Macaluso, F. P., Asara, J. M., et al. (2013). Centriole distal appendages promote membrane docking, leading to cilia initiation. Genes Dev. 27, 163-168. doi: 10.1101/gad.207043.112

Thomas, J., Morlé, L., Soulavie, F., Laurencon, A., Sagnol, S., and Durand, B. (2010). Transcriptional control of genes involved in ciliogenesis: a first step in making cilia. Biol. Cell 102, 499-513. doi: 10.1042/BC20100035

Tissir, F., Qu, Y., Montcouquiol, M., Zhou, L., Komatsu, K., Shi, D., et al. (2010). Lack of cadherins Celsr2 and Celsr3 impairs ependymal ciliogenesis, leading to fatal hydrocephalus. Nat. Neurosci. 13, 700-707. doi: 10.1038/nn.2555

Veleri, S., Manjunath, S. H., Fariss, R. N., May-Simera, H., Brooks, M., Foskett, T. A., et al. (2014). Ciliopathy-associated gene Cc2d2a promotes assembly of subdistal appendages on the mother centriole during cilia biogenesis. Nat. Commun. 5:4207. doi: 10.1038/ncomms5207

Wodarczyk, C., Rowe, I., Chiaravalli, M., Pema, M., Qian, F., and Boletta, A. (2009). A novel mouse model reveals that polycystin-1 deficiency in ependyma and choroid plexus results in dysfunctional cilia and hydrocephalus. PLoS One 4:e7137. doi: 10.1371/journal.pone.0007137

Yoshimura, K., Kawate, T., and Takeda, S. (2011). Signaling through the primary cilium affects glial cell survival under a stressed environment. Glia 59, 333-344. doi: 10.1002/glia.21105

Conflict of Interest Statement: The authors declare that the research was conducted in the absence of any commercial or financial relationships that could be construed as a potential conflict of interest.

Received: 29 September 2014; paper pending published: 13 October 2014; accepted: 27 January 2015; published online: 12 February 2015.

Citation: Narita K and Takeda S (2015) Cilia in the choroid plexus: their roles in hydrocephalus and beyond. Front. Cell. Neurosci. 9:39. doi: 10.3389/fncel.2015.00039 This article was submitted to the journal Frontiers in Cellular Neuroscience.

Copyright (C) 2015 Narita and Takeda. This is an open-access article distributed under the terms of the Creative Commons Attribution License (CC BY). The use, distribution and reproduction in other forums is permitted, provided the original author(s) or licensor are credited and that the original publication in this journal is cited, in accordance with accepted academic practice. No use, distribution or reproduction is permitted which does not comply with these terms. 